\title{
Metastatic thyroid carcinoma mimicking as a primary neoplasia of the kidney: A case report
}

\author{
ANTONIO IENI $^{1}$, GUIDO FADDA ${ }^{1}$, GIUSEPPE ALARIO ${ }^{2}$, ANTONELLA PINO ${ }^{3}$, \\ VINCENZO FICARRA $^{2}$, GIANLORENZO DIONIGI ${ }^{3}$ and GIOVANNI TUCCARI ${ }^{1}$ \\ ${ }^{1}$ Pathology Section, ${ }^{2}$ Urology Section and ${ }^{3}$ Endocrine and Minimally Invasive Surgery Section, \\ Department of Human Pathology in Adulthood and Childhood 'Gaetano Barresi', \\ University Hospital G. Martino, University of Messina, I-98125 Messina, Italy
}

Received June 13, 2021; Accepted July 19, 2021

DOI: $10.3892 / \mathrm{mco} .2021 .2430$

\begin{abstract}
The unfavorable behavior of primary thyroid carcinoma (PTC) has been revealed by the hematogenous distant metastases in $\sim 20-25 \%$ of cases with frequent localizations in lungs and bones, but infrequently in kidney. A 69-year-old male patient was admitted to Department of Human Pathology in Adulthood and Childhood 'G. Barresi', University Hospital G. Martino, (Messina, Italy) for an incidentally detected parenchymal mass involving the right kidney. A partial nephrectomy was done; at the post-surgical examination, a large nodular grayish mass was documented. Microscopically, a diffuse proliferation with solid/follicular pattern with some colloid-filled spaces was appreciable. An intense immunopositivity was revealed for thyroglobulin, thyroid transcription factor-1 (TTF-1), paired-box gene 8 (PAX-8) and cytokeratin 7, while CD10 and renal cell carcinoma marker were negative. A diagnosis of the metastatic thyroid follicular carcinoma localized in the kidney was made. At ultrasound examination, a hyperechoic mass extending from the left thyroid lobe to the isthmus, to which TIR4 diagnostic category according to the Italian reporting system for thyroid cytology was attributed. After thyroid surgical procedure, the final diagnosis of primitive differentiated follicular thyroid carcinoma with foci of poorly differentiated component was made.
\end{abstract}

Correspondence to: Professor Giovanni Tuccari, Pathology Section, Department of Human Pathology in Adulthood and Childhood 'Gaetano Barresi', University Hospital G. Martino, University of Messina, Pad. D, Street C. Valeria 1, I-98125 Messina, Italy

E-mail: tuccari@unime.it

Key words: metastasis, kidney, partial nephrectomy, differentiated thyroid carcinoma, radio metabolic therapy

\section{Introduction}

The risk factors correlated with recurrence of primary thyroid carcinoma (PTC) are being $>45$ years, primary tumor size $>40 \mathrm{~mm}$, widespread invasion, multifocality, positive resection margin, lymph node and distant metastases at diagnosis $(1,2)$. The aggressive behavior of PTC has been mainly revealed by the hematogenous occurrence of distant metastases in $20-25 \%$ of cases, with more frequent localizations to the lungs and bones $(1,2)$, while other unusual sites are represented by skin and skull base (3-5). In addition, the renal metastatic involvement from PTC represents a very rare event, accounting for $<5 \%$ of cases $(6,7)$; in fact, only 18 cases have been reported in the English literature, although an additional 30 cases have been reported in the Japanese literature (8-14). The main PTC able to determine vascular invasion and metastasize to the kidney is represented by differentiated follicular carcinoma, followed by Hurthle cell as well as poorly differentiated carcinomas, since papillary carcinoma usually presents a lymphatic metastatic spread $(2,8,10)$. Unfortunately, distant metastases may appear as the initial symptom of PTC with the thyroid function remaining unaltered (13-16); hence, it is not surprising that metastases occurring in unusual sites may be misdiagnosed as primary cancer until the post-surgical pathological analysis has been carried out revealing the metastatic nature from PTC. In light of these conditions, an interesting case report is presented herein with a case of a large single renal metastasis from thyroid follicular carcinoma with some poorly differentiated associated foci. It is important to have a fundamental knowledge of the characteristics of PTC, especially in thus advanced stage, since these tumors often require multidisciplinary management using multiple imaging and treatment modalities.

\section{Case report}

A 69-year-old man was admitted to Department of Human Pathology in Adulthood and Childhood 'G. Barresi', University Hospital G. Martino, (Messina, Italy) in January 2021 suffering from backache and spine discomfort and was consequently subjected to abdominal computed tomography (CT). The CT examination incidentally detected a parenchymal renal mass involving the kidney, represented by a large $7.5 \times 6.0 \mathrm{~cm}$ mass 
on the right kidney (Fig. 1), which included some minor not homogeneous areas referred to hemorrhage or necrosis. No additional risk factors for renal pathology were found.

Preoperatively, the patient had a body mass index (BMI) of 27 , he was fully active and without relevant co-morbidities (age-adjusted Charlson score $=0$ ). The maximum tumor size was $6 \mathrm{~cm}$ and the PADUA nephrometry score was 10 (lesion with a high risk of complications if partial nephrectomy is planned) (17). Preoperative thoracic and abdominal CT scan was negative for lymph node involvement and/or distant metastases. Hence, the clinical stage of the patient was determined to be cT1bN0M0. The contralateral kidney was normal. Preoperative laboratory tests showed normal renal function; in detail, renal function was tested using the Erythrocyte Glomerular Filtration Rate (eGFR) starting from the creatinine level. However, the international guidelines (EAU Guidelines) (18) do not consider it necessary to perform a kidney scan in the preoperative evaluation of candidate patients for partial nephrectomy. The American Society of Anesthesiologists (ASA) score of the patient was category III (18). According to the international guidelines, an elective partial nephrectomy was performed on the patient according to the guidelines on Renal Cell Carcinoma 2021 edition (18).

In detail, a flank incision and a retroperitoneal open approach was performed. After identification of the main renal artery, the kidney was extensively mobilized until easy access to the tumor from all sides was achieved. All the perirenal fat tissue was removed except for that located on the top of the tumor. The tumor was demarcated and then the main artery clamped (warm ischemia). The renal capsule was incised very close to the tumor and a plan between the healthy parenchyma and the tumor capsule was developed performing a simple enucleation. The inner defect was closed with a running Monocryl 4-0 suture (Johnson \& Johnson Medical N.V.) preloaded with a Absolok clip (Johnson \& Johnson Medical N.V.). The Monocryl was at the end brought outside through the parenchyma and secured with a second Absolok clip. Through the sliding clip technique, the right tension was brought on this suture. Cortical renorrhaphy was performed using interrupted 2-0 (26 mm needle) polyfilament sutures placed at intervals of $1 \mathrm{~cm}$ using the sliding-clip technique with Absolok ${ }^{\circledR}$ (Johnson \& Johnson Medical N.V.) clips (19). The clamp on the main artery was removed after $14 \mathrm{~min}$. The heamostatic agent Floseal ${ }^{\circledR}$ (Baxter International) was applied on the cortical defect to complete the hemostasis. No intraoperative complication was observed. The estimated blood loss was $200 \mathrm{ml}$. Neither intra-operative nor post-operative blood transfusion were needed. No post-operative complications were recorded, and the patient was discharged in 7 postoperative day (POD) with normal laboratory tests.

Grossly, the partial renal resection documented a large nodular grayish mass of $70 \times 60 \times 35 \mathrm{~mm}$ diameter protruding to the capsule (Fig. 2), but presenting inside some regressive cystic black-reddish portions. No invasion of perirenal adipose tissue, neither involvement of renal vessels nor the adrenal gland were appreciable. A slight centimetric portion of uninvolved renal tissue circumscribed the neoplastic mass.

All samples of the renal lesion were fixed in $10 \%$ neutral formalin for 24-36 $\mathrm{h}$ at room temperature, embedded in paraffin at $56^{\circ} \mathrm{C}$, and then cut into $5-\mu \mathrm{m}$ thick serial sections in order to perform routine hematoxylin/eosin histological staining. For the immunohistochemical procedure pparallel sections were cut and mounted on silane-coated glass, then dewaxed in xylene and rehydrated in graded ethanol. Histologically, the neoplasia exhibited a solid and or follicular pattern in which some colloid-filled spaces were appreciable (Fig. 3A); the adjacent renal parenchyma was lined by a fibrous capsule, but lymphovascular invasion was noted without satellite neoplastic foci. Finally, surgical as well as ureteral margins were all negative for the tumor. Taking into account that the documented renal neoplasia could be attributable to thyroid follicular-like renal carcinoma, as previously reported (19), immunohistochemical procedures were performed. Immunohistochemistry was performed using the automated Ventana BenchMark ULTRA platform with Cell Conditioning 1 for $64 \mathrm{~min}$, pre-peroxidase inhibition and primary antibody incubation for $16 \mathrm{~min}$ at $37^{\circ} \mathrm{C}$. The OptiView DAB IHC Detection kit (Ventana Medical Systems, Inc.) was used to detect protein expression of the following primary antibodies: thyroglobulin (TG; 1:300; cat. no. 760-2671; Roche Diagnostics), Thyroid transcription factor-1 (TTF-1; 1:300; cat. no 790-4398; Roche Diagnostics), cytokeratin 7 (CK 7; 1:500; cat. no. 790-4462; Roche Diagnostics), Paired-box gene 8 (PAX-8; 1:200; cat. no. 760-4618; Roche Diagnostics), CD10 (1:200; cat. no. 790-4506; Roche Diagnostics), carbonic anhydrase IX (CAIX; 1:300; cat. no. 760-6080; Roche Diagnostics), $\alpha$-methylacyl-CoA racemase (AMACR; 1:250; cat. no. 790-6011; Roche Diagnostics) and renal cell carcinoma (RCC; 1:100; cat. no. 760-4273; Roche Diagnostics). Finally, all slides were counterstained with Hematoxylin II (Ventana Medical Systems, Inc.) and Bluing Reagent (Ventana Medical Systems, Inc.) for $4 \mathrm{~min}$ at room temperature. In detail, positive immunostaining was revealed for TG (Fig. 3B), TTF-1 (Fig. 3C), CK 7, PAX-8 while CD10 (Fig. 3D), CAIX, AMACR and RCC marker were constantly negative.

Consequently, morphological and immunohistochemical findings strongly supported the metastatic nature of the renal tumor originating from a thyroid follicular carcinoma and hence, the patient underwent an ultrasound examination. A large solitary hyperechoic mass $(5.8 \times 4.2 \mathrm{~cm})$, with some focal anechoic irregular areas, extending from the left thyroid lobe to the isthmus was revealed. Fine needle aspiration cytology (FNAC) of the nodule, stained with May-Grünwald-Giemsa stain, documented numerous sheets and clusters, sometimes solid or microfollicular-patterned, with oval or round nuclei presenting some degree of pleomorphism, but without nuclear inclusions (Fig. 4A and B); a TIR4 according to the Italian reporting system for thyroid cytology (20) was attributed to the lesion and the patient was admitted to the Endocrine and Minimally Invasive Surgery Section, University Hospital G. Martino, University of Messina (Messina, Italy).

A total thyroidectomy was performed: the left lobe weighed $53.3 \mathrm{~g}$ and it was fully substituted by a firm, white-grayish nodule of $6 \times 4 \times 4 \mathrm{~cm}$, joined to the isthmus of $1.0 \times 0.7 \mathrm{~cm}$ with a solid sclero-calcific area; the right lobe weighed only $9.0 \mathrm{~g}$ and it presented a multinodular goiter appearance with dimensions of $5.0 \times 2.0 \times 1.5 \mathrm{~cm}$. In the perithyroidal adipose tissue, only 2 reactive lymph nodes were isolated. The histopathological examination of the surgical samples confirmed the neoplastic nature of the nodule, mainly organized in microfollicular struc- 


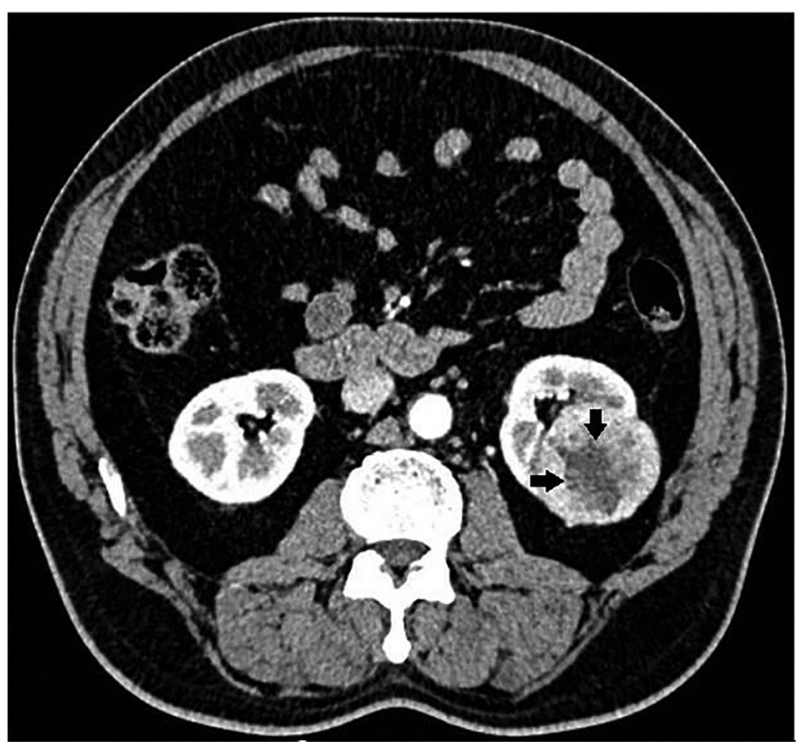

Figure 1. Imaging of neoplasm. Computed tomography revealed a large mass (70x60 mm) with medial-inferior renal location, without any involvement of hilar vessels. Black arrows indicate intralesional necrosis.

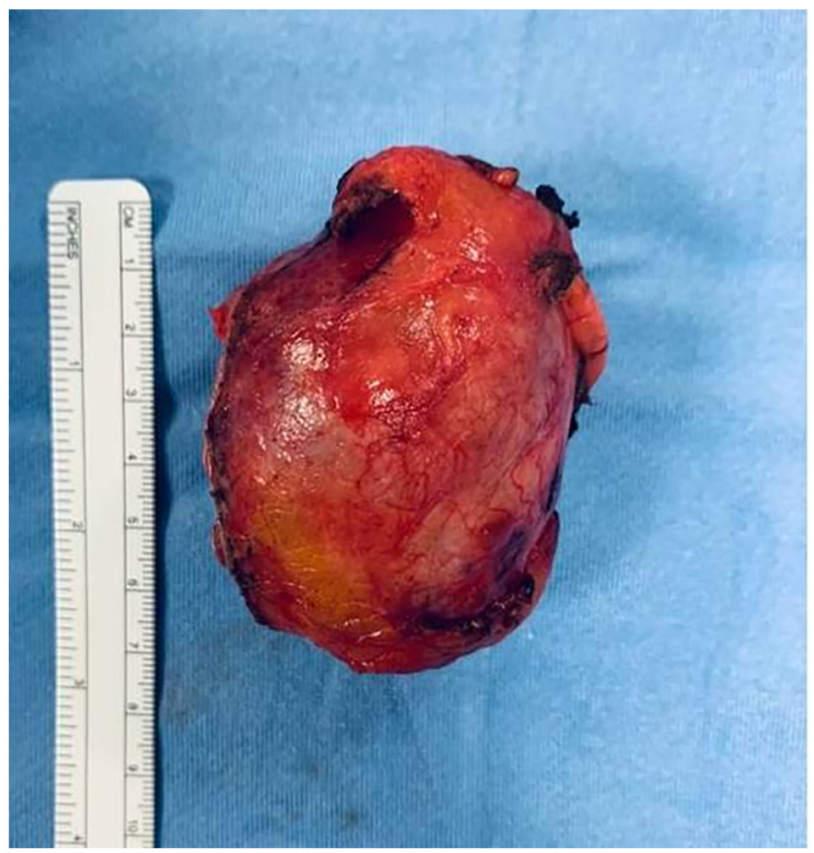

Figure 2. Macroscopic aspect of the tumor. The renal resection documented a large well-circumscribed mass with some regressive black-reddish portions.

tures, although an insular, solid and less differentiated pattern occurred in $\sim 30 \%$ of thyroid carcinoma (Fig. $5 \mathrm{~A}$ and B); partial invasion of fibrous capsule as well as of 4 invasive vessels neoplastic foci were recorded. Immunohistochemistry confirmed the positivity for thyroglobulin and TTF-1, with no staining for galectin-3, p53, CDX2 and BRAF V600E; the growth fraction, analyzed by Ki67 labeling index was $15 \%$. A final diagnosis of primitive differentiated follicular thyroid carcinoma (pT3aN0 according to TNM/AJCC 2017) (21), with poorly differentiated foci $(30 \%)$ infiltrating the capsule and angioinvasion was made.
On the last follow-up (July 2021) the patient was in a good overall condition and computed tomography control revealed no tumour recurrence in either the renal or thyroid sites.

\section{Discussion}

The prognosis of DTC is promising, with a 10 -year survival rate of $80-95 \%$; however, this is reduced to $50 \%$ when metastases are present (22). Furthermore, age at diagnosis is a significant prognostic indicator for the risk of recurrence and death, particularly in patients $>40$ years of age (22). It is well known that distant metastasis in DTC can be divided into 2 groups: i) Distant metastasis as the initial presenting diagnosis; and ii) distant metastasis after the initial treatment of thyroid cancer (23). The incidence of distant metastasis after the initial treatment of DTC is between $7-23 \%$, and the frequency of diagnosed DTC presenting initially with metastatic disease ranges from $1-9 \%$ (23). In particular, distant metastasis in FTC have been reported in $6-20 \%$ of cases, and the survival rates range from $31-43 \%$ (22). In light of these sporadic reports, the novelty of the current case is greatly substantiated by the unspecific symptoms, focusing the clinical attention on the lumbar region; in other words, the present report may be defined as an 'incidentaloma' due to the occurrence of renal metastatic deposit prior to the correct diagnosis of a primitive DTC. Consequently, the morphological definition of the secondary lesion guided further diagnostic process towards the thyroid gland. However, due to a lack of cases and randomized studies, there is no consensus for the management of distant metastasis in patients with DTC, even if a complete resection of DTC metastasis remains a good option, as has been suggested elsewhere (24-26).

In contrast to high incidence of PTC, distant metastases of this thyroid histotype are rarely seen and generally presented as a single case report $(14,15)$; however, follicular carcinomas usually represent a neoplastic aggressive entity able to metastasize more frequently compared with papillary carcinoma by haematogeneous spread to distant sites, such as lung, bone, but seldom skin and kidney $(1,3-5,13,14)$. Commonly, in unusual renal localization, thyroid metastases results as multiple/or bilateral nodules, probably related to the presence of venous vascular structures between the thyroid and kidney $(6,7)$. As a general rule, when thyroid tumors metastasize to the kidney, lesions appear multifocal and bilateral (12); nevertheless, it has been reported previously in case reports as a solitary renal mass $(11,12)$. However, a renal solitary neoplastic mass is rarely attributed to thyroid metastasis, mainly when a general check of patients has not been performed. Although the pathological preoperative diagnosis of renal tumors by fine needle and core needle biopsy is not requested anymore, the presurgical awareness of nature of the mass should be considered as a useful approach in some selected cases. In addition, metastatic thyroid tumor in the kidney can be difficult to diagnose on cytologic smears since the nuclear features are easily suggestive for diagnosis only when papillary histotype is the primary cancer.

A crucial point to define the nature of a single renal mass, with a histological appearance of follicular carcinoma, is 

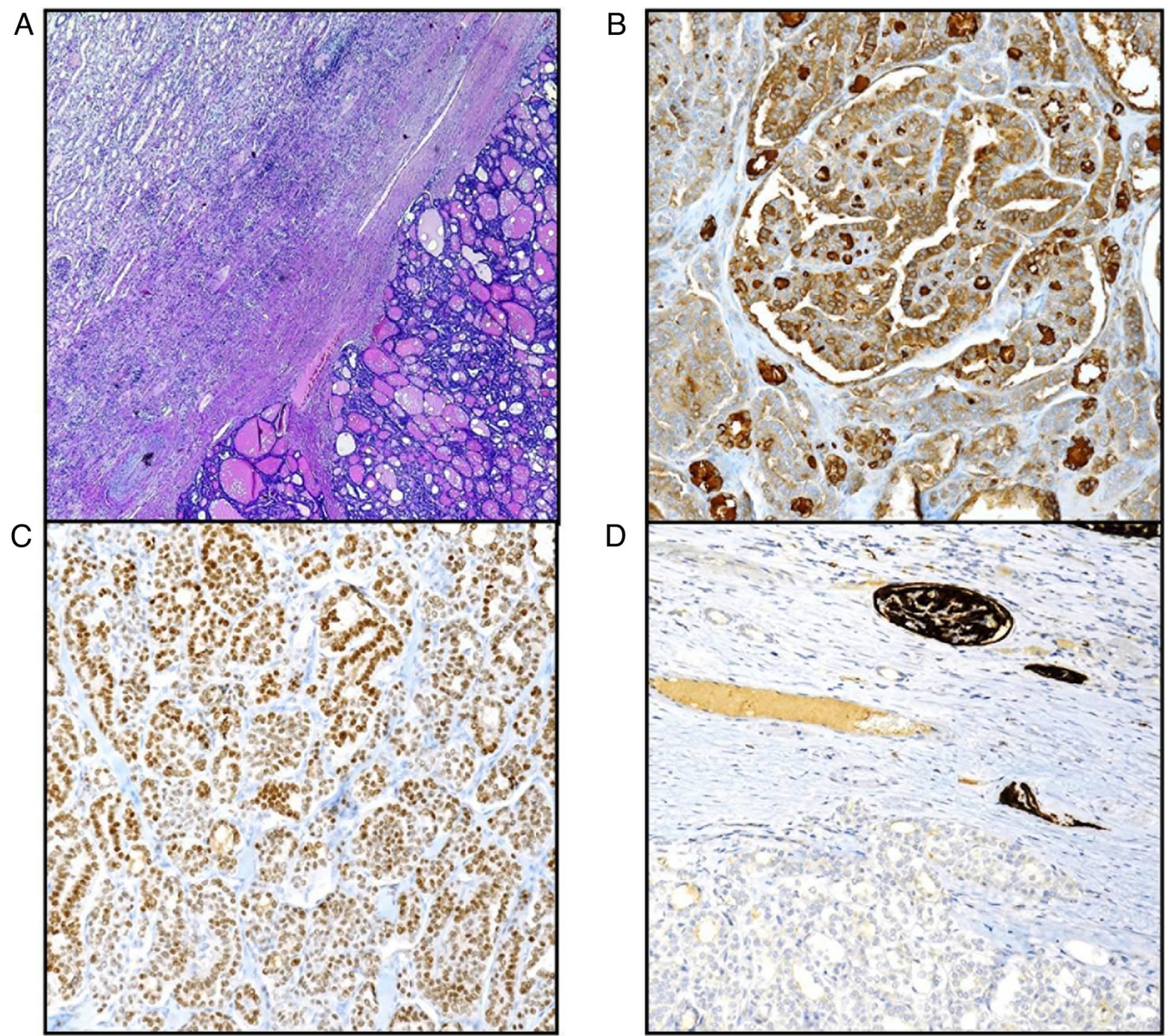

Figure 3. Histological and immunohistochemical findings of the renal specimen. (A) Follicular pattern was evident by hematoxylin and eosin staining (magnification, x120). Positive immunostaining was for (B) thyroglobulin (magnification, x240), (C) TTF-1 (magnification, x120). (D) CD10 was constantly negative (magnification, x240). TTF-1, thyroid transcription factor-1.
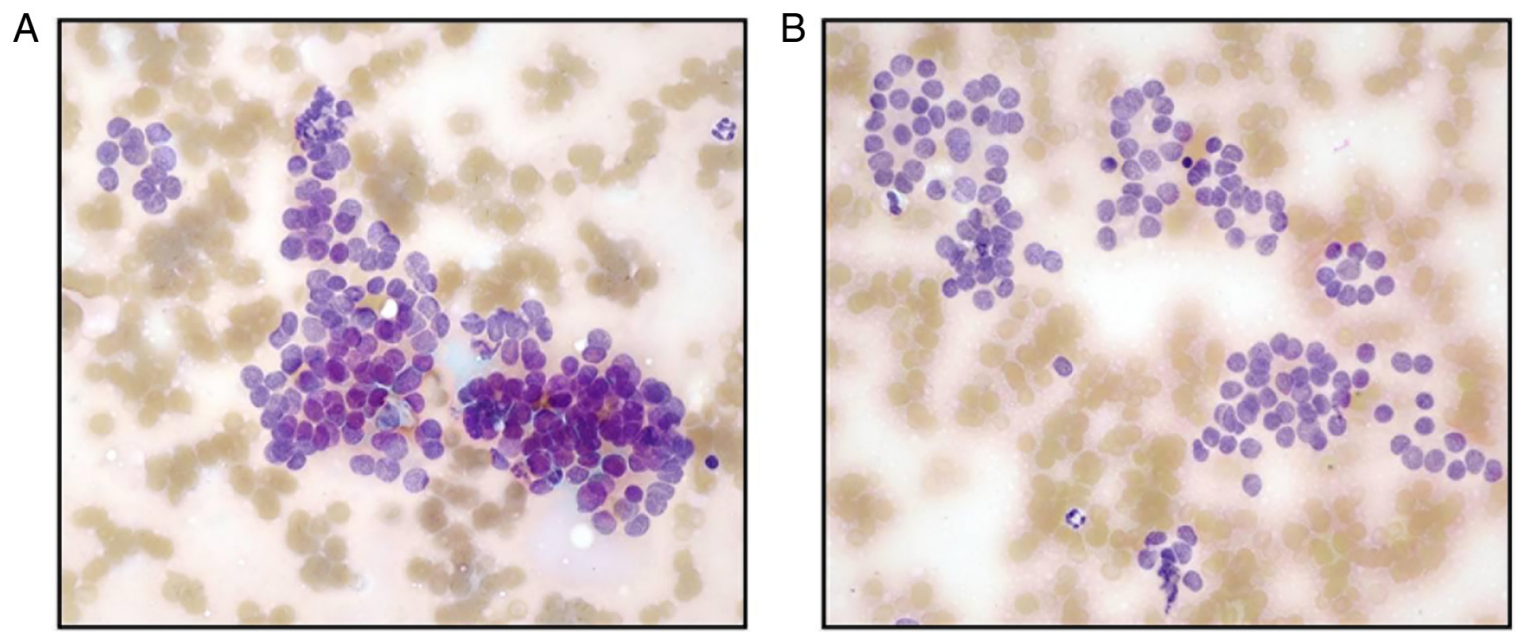

Figure 4. Cytological smears. (A) In thyroid nodule solid sheets of follicular well differentiated elements were appreciable (May-Grúnwald-Giemsa; magnification, x400); (B) in other microscopic fields, follicular-patterned structures with slight nuclear pleomorphism without any nuclear inclusions (May-Grünwald-Giemsa, magnification, x400).

to accurately differentiate the thyroid-like follicular renal carcinoma (TLFC) which represents a peculiar pattern in the renal tumor, firstly described 15 years ago (27); generally, the majority of TLFC cases were low grade with of 

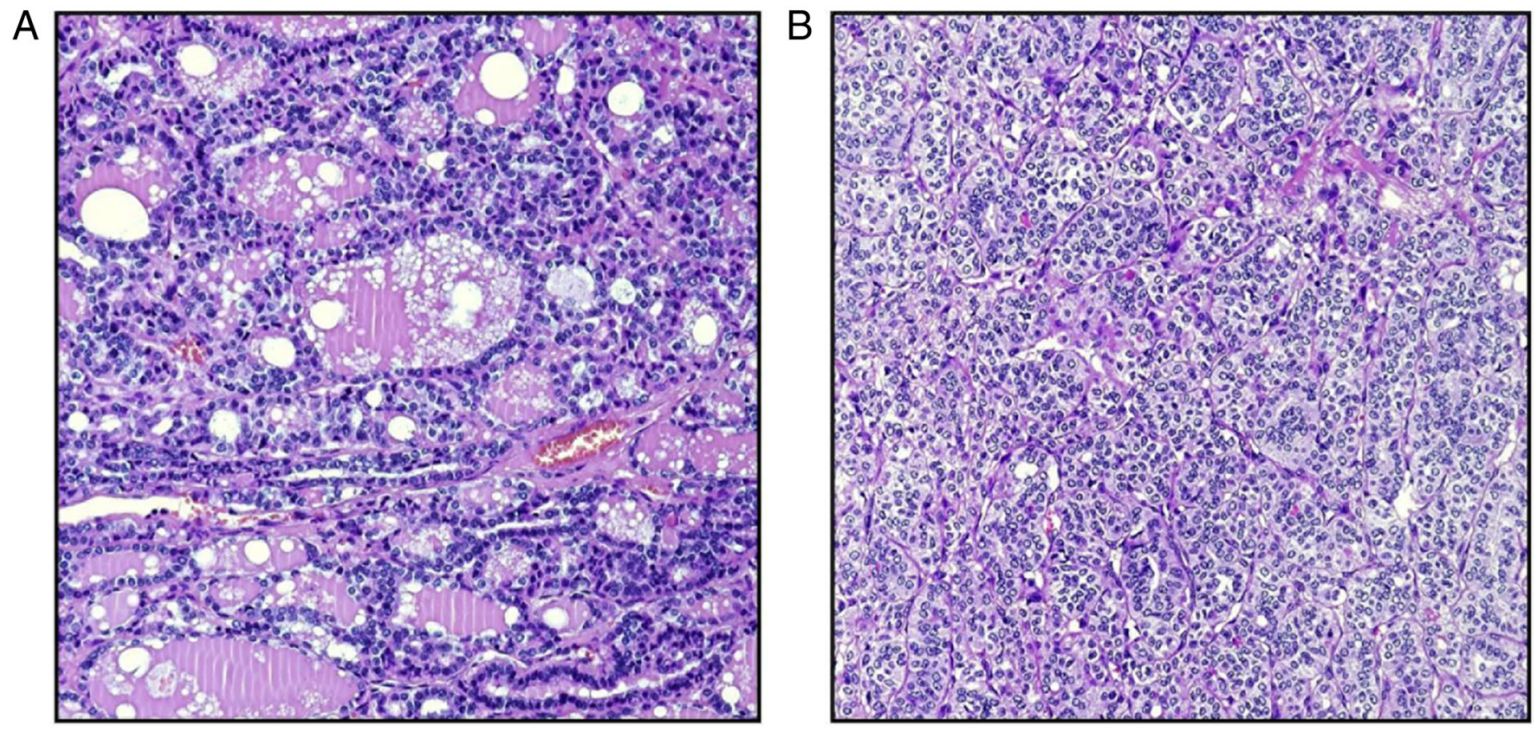

Figure 5. Histopathological analysis. The neoplasm revealed a (A) greater extension of microfollicular structures, while (B) solid and less differentiated areas were elsewhere appreciable (hematoxylin and eosin staining; magnification, x240).

indolent course, even if renal hilar lymph node and another widespread retroperitoneal lymph node or lung metastases have been described (27). Histologically, the morphology of TLFC is quite identical to the findings of the present case, with widespread microfollicles and macrofollicles containing abundant colloid-like material, bearing a striking resemblance to follicular carcinoma of the thyroid gland. However, thyroidisation of the kidney has already been reported in patients with chronic pyelonephritis, as well as with end-stage renal disease; in this latter entity, the renal tissue shows a thyroid-like feature characterized by atrophic distal tubules and colloid-like hyaline casts.

In this diagnostic challenge, a relevant important role should be attributed to immunohistochemistry since while renal cell follicular-like carcinomas are constantly immunostained for CD10, RCC, vimentin, metastatic thyroid carcinoma to the kidney exhibits a specific staining for TTF-1 and thyroglobulin, as found in previous studies $(11,20,28)$, but also in the case in the present study.

Management of renal metastasis from thyroid carcinoma, firstly includes surgical procedure consisting in radical nephrectomy or laparoscopic partial nephrectomy $(6,12,29)$; these approaches should be combined with high dose radio-iodine therapies (12). The rationale of surgery to remove renal metastases is based on the need to perform a neoplastic debulking, obtaining a reduction of the tumor burden that have to be successively treated by radioactive iodine treatment. In fact, after surgical treatment the radio metabolic therapy may allow clinical remission and an improved 10-year overall survival rate even in cases with additional future bone and lung metastasis $(6,30)$.

Hence, in conclusion, the therapeutic surgical strategy used in the reported case represents a good and promising approach. In fact, only the morphological analysis of metastatic renal deposits addressed the correct investigative approach concerning the primary neoplastic origin from the thyroid. After the cytological diagnostic validation, total thyroidectomy with regional lymph node dissection appears to be the most appropriate treatment, followed by radioablative therapy to remove potential residual disease or metastases. Finally, when neoplastic renal mass of unidentified origin is revealed in patients with unspecific symptoms, the presence of occult undiagnosed thyroid carcinoma should be considered.

\section{Acknowledgements}

Not applicable.

\section{Funding}

No funding was received.

\section{Availability of data and materials}

The datasets used and/or analyzed during the present study are available from the corresponding author on reasonable request.

\section{Authors' contributions}

AI and GT designed the study and wrote the manuscript. GA, AP, VF and GD performed the surgical procedures. AI, GF and GT performed the morphology. AI, GF and GT wrote the manuscript. All the authors read and approved the final manuscript.

\section{Ethics approval and consent to participate}

Not applicable.

\section{Patient consent for publication}

Written informed consent was obtained from the patient for publication of this case report. 


\section{Competing interests}

The authors declare that they have no competing interests.

\section{References}

1. D'Avanzo A, Ituarte P, Treseler P, Kebebew E, Wu J, Wong M, Duh QY, Siperstein AE and Clark OH: Prognostic scoring systems in patients with follicular thyroid cancer: A comparison of different staging systems in predicting the patient outcome. Thyroid 14: 453-458, 2004.

2. Iwai H, Ohno Y, Ito H, Kiyokawa T and Aoki N: Renal rupture associated with a poorly differentiated follicular thyroid carcinoma metastasizing to the thigh muscle, lung and kidney. Intern Med 44: 848-852, 2005.

3. Camacho V, Rodríguez-Revuelto A, Flotats A, Duch J, Artigas C, Carrió I and Estorch M: Skin metastasis of follicular thyroid carcinoma. Eur J Nucl Med Mol Imaging 37: 1237, 2010.

4. Matsuno A, Katakami H, Okazaki R, Yamada S, Sasaki M Nakaguchi H, Yamada SM, Hoya K, Murakami M, Yamazaki K, et al: Skull base metastasis from follicular thyroid carcinoma-two case reports. Neurol Med Chir (Tokyo) 50: 421-425, 2010

5. Song HJ, Xue YL, Xu YH, Qiu ZL and Luo QY: Rare metastases of differentiated thyroid carcinoma: Pictorial review. Endocr Relat Cancer 18: R165-R174, 2011.

6. Cochetti G, Puxeddu E, Zingaro MD, D'Amico F, Cottini E, Barillaro F and Mearini E: Laparoscopic partial nephrectomy of thyroid cancer metastasis: Case report and review of the literature. Onco Targets Ther 6: 355-360, 2013.

7. Kumar A, Nadig M, Patra V, Srivastava DN, Verma K and Bal CS: Adrenal and renal metastases from follicular thyroid cancer. Br J Radiol 78: 1038-1041, 2005.

8. Moudouni SM, En-Nia I, Rioux-Leclerq N, Manunta A, Guille F and Lobel B: Follicular carcinoma of the thyroid metastasis to the kidney nine years after resection of the primary tumor. Ann Urol (Paris) 36: 36-37, 2002.

9. Liou MJ, Lin JD, Chung MH, Liau CT and Hsueh C: Renal metastasis from papillary thyroid microcarcinoma. Acta Otolaryngol 125: 438-442, 2005.

10. Djekidel M, Gordon M, Shah RB, Gross MD and Avram A: Renal metastasis from Hurthle cell thyroid carcinoma and its evaluation with hybrid imaging. Thyroid 20: 429-433, 2010.

11. Xu H, Zeng W and Tang Y: Metastatic thyroid follicular carcinoma presenting as a primary renal tumor. Intern Med 51: 2193-2196, 2012.

12. Nath V, Baliga M, Lewin J, Souza F and Akhtar I: Follicular thyroid carcinoma metastatic to the kidney: Report of a case with cytohistologic correlation. Case Rep Pathol 2015: 701413, 2015.

13. Cai DM, Wang HY, Jiang Y, Parajuly SS, Tian YE, Ma BY, Li YZ, Song B and Luo Y: Primary follicular thyroid carcinoma metastasis to the kidney and widespread dissemination: A case report. Oncol Lett 11: 3293-3297, 2016.

14. Zhou C, Urbauer DL, Fellman BM, Tamboli P, Zhang M, Matin SF, Wood CG and Karam JA: Metastases to the kidney: A comprehensive analysis of 151 patients from a tertiary referral centre. BJU Int 117: 775-782, 2016.

15. Gezer E, Selek A, Tarkun İ, Cantürk Z and Çetinarslan B Papillary thyroid carcinoma presenting as a primary renal tumor with multiple pulmonary and bone metastases: A case report. J Med Case Rep 13: 95, 2019.

16. Sampson E, Brierley JD, Le LW, Rotstein L and Tsang RW: Clinical management and outcome of papillary and follicular (differentiated) thyroid cancer presenting with distant metastasis at diagnosis. Cancer 110: 1451-1456, 2007.
17. Ficarra V, Novara G, Secco S, Macchi V,Porzionato A, De Caro R and Artibani W: Preoperative aspects and dimensions used for an anatomical (PADUA) classification of renal tumours in patients who are candidates for nephron-sparing surgery. Eur Urol 56: 786-793, 2009

18. Bedke J, Albiges L, Capitanio U, Giles RH, Hora M, Lam TB, Ljungberg B, Marconi L, Klatte T, Volpe A, et al: The 2021 updated European association of urology guidelines on renal cell carcinoma: Immune checkpoint inhibitor-based combination therapies for treatment-naive metastatic clear-cell renal cell carcinoma are standard of care. Eur Urol: May 29, 2021 (Epub ahead of print).

19. Rossanese M,Crestani A, Giannarini G,Calandriello M, Alario G, Simonato A and Ficarra V: Absolok ${ }^{\circledR}$ versus Hem-o-Lok ${ }^{\circledR}$ clips for renorrhaphy during partial nephrectomy for parenchymal renal tumors. Minerva Urol Nephrol 72: 91-98, 2020.

20. Poller DN, Baloch ZW, Fadda G, Johnson SJ, Bongiovanni M, Pontecorvi A and Cochand-Priollet B: Thyroid FNA: New classifications and new interpretations. Cancer Cytopathol 124: 457-466, 2016.

21. Amin MB, Edge S, Greene F, Byrd DR, Brookland RK, Washington MK, Gershenwald JE, Compton CC, Hess KR, Sullivan DC, et al (eds): American Joint Committee on Cancer Cancer Staging Manual. 8th edition. Springer International Publishing, Manhattan, New York, NY, 2017.

22. Grønlund MP, Jensen JS, Hahn CH, Grønhøj C and von Buchwald C: Risk factors for recurrence of follicular thyroid cancer: A systematic review. Thyroid: Jul 5, 2021 (Epub ahead of print).

23. Veschi V, Verona F, Lo Iacono M, D'Accardo C, Porcelli G, Turdo A, Gaggianesi M, Forte S, Giuffrida D, Memeo L and Todaro M: Cancer stem cells in thyroid tumors: From the origin to metastasis. Front Endocrinol (Lausanne) 11: 566, 2020.

24. Kunadharaju R, Goyal G, Rudraraju A and Silberstein PT: New treatment options for metastatic thyroid cancer. Fed Pract 32 (Suppl 7): 21S-26S, 2015.

25. Pacini F, Basolo F, Bellantone R, Boni G, Cannizzaro MA, De Palma M, Durante C, Elisei R, Fadda G, Frasoldati A, et al: Italian consensus on diagnosis and treatment of differentiated thyroid cancer: Joint statements of six Italian societies. J Endocrinol Invest 41: 849-876, 2018.

26. Metere A, Aceti V and Giacomelli L: The surgical management of locally advanced well-differentiated thyroid carcinoma: Changes over the years according to the AJCC 8th edition cancer staging manual. Thyroid Res 12: 10, 2019.

27. Jung SJ, Chung JI, Park SH, Ayala AG and Ro JY: Thyroid follicular carcinoma-like tumor of kidney: A case report with morphologic, immunohistochemical, and genetic analysis. Am J Surg Pathol 30: 411-415, 2006.

28. Cimino-Mathews A, Sharma R and Netto GJ: Diagnostic use of PAX8, CAIX, TTF-1, and TGB in metastatic renal cell carcinoma of the thyroid. Am J Surg Pathol 35: 757-761, 2011.

29. Pak H, Gourgiotis L, Chang WI, Guthrie LC, Skarulis MC, Reynolds JC, Merino MJ, Schrump DS, Libutti SK, Alexander HR Jr and Sarlis NJ: Role of metastasectomy in the management of thyroid carcinoma: The NIH experience. J Surg Oncol 82: 10-18, 2003.

30. Durante C, Haddy N, Baudin E, Leboulleux S, Hartl D, Travagli JP, Caillou B, Ricard M, Lumbroso JD, De Vathaire F and Schlumberger M: Long-term outcome of 444 patients with distant metastases from papillary and follicular thyroid carcinoma: Benefits and limits of radioiodine therapy. J Clin Endocrinol Metab 91: 2892-2899, 2006.

This work is licensed under a Creative Commons Attribution-NonCommercial-NoDerivatives 4.0 International (CC BY-NC-ND 4.0) License. 